

\title{
Decision support with ill-known criteria in the collaborative supply chain context
}

Romain Guillaume, Guillaume Marquès, Caroline Thierry, Didier Dubois

\section{To cite this version:}

Romain Guillaume, Guillaume Marquès, Caroline Thierry, Didier Dubois. Decision support with ill-known criteria in the collaborative supply chain context. Engineering Applications of Artificial Intelligence, 2014, vol. 36, pp. 1-11. 10.1016/j.engappai.2014.06.013 . hal-01153898

\section{HAL Id: hal-01153898 \\ https://hal.science/hal-01153898}

Submitted on 20 May 2015

HAL is a multi-disciplinary open access archive for the deposit and dissemination of scientific research documents, whether they are published or not. The documents may come from teaching and research institutions in France or abroad, or from public or private research centers.
L'archive ouverte pluridisciplinaire HAL, est destinée au dépôt et à la diffusion de documents scientifiques de niveau recherche, publiés ou non, émanant des établissements d'enseignement et de recherche français ou étrangers, des laboratoires publics ou privés. 


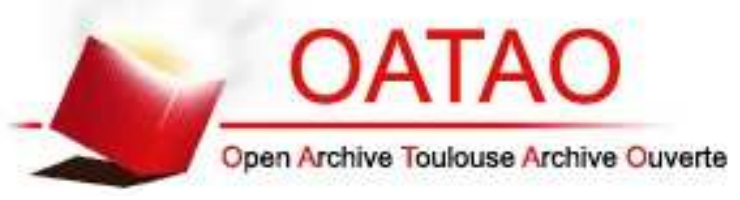

\section{Open Archive TOULOUSE Archive Ouverte (OATAO)}

OATAO is an open access repository that collects the work of Toulouse researchers and makes it freely available over the web where possible.

This is an author-deposited version published in : http://oatao.univ-toulouse.fr/ Eprints ID : 13200

To link to this article : DOI:10.1016/j.engappai.2014.06.013

URL : http://dx.doi.org/10.1016/j.engappai.2014.06.013

To cite this version : Guillaume, Romain and Marques, Guillaume and Thierry, Caroline and Dubois, Didier Decision support with ill-known criteria in the collaborative supply chain context. (2014) Engineering Applications of Artificial Intelligence, vol. 36 . pp. 1-11. ISSN 0952-1976

Any correspondance concerning this service should be sent to the repository administrator: staff-oatao@listes-diff.inp-toulouse.fr 


\title{
Decision support with ill-known criteria in the collaborative supply chain context
}

\author{
R. Guillaume ${ }^{\mathrm{a}, *}$, G. Marques $^{\mathrm{b}}$, C. Thierry $^{\mathrm{a}}$, D. Dubois ${ }^{\mathrm{a}}$ \\ a IRIT - Université de Toulouse, Université Paul Sabatier, 118 Route de Narbonne, F-31062 Toulouse Cedex 9, France \\ ${ }^{\mathrm{b}}$ FAYOL-EMSE, CNRS-UMR5600, EVS, Ecole Nationale Supérieure des Mines, F-42023 Saint-Etienne, France
}

Keywords:

Sequential decision

Supply chain collaboration

Possibility theory

Decision tree

Hurwicz criteria

\begin{abstract}
A B S T R A C T
In the field of Supply Chain Risk Management, the attitude of managers toward risk affect the tactical decision-making process in collaborative supply chains under an uncertain environment, concerning especially capacity levels, lot-sizing rules, purchasing strategies, production scheduling,..., etc. The issue can be formulated as a sequential decision problem under uncertainty where the customer decisions affect the decisions made by the supplier. In this paper we deal with two kinds of uncertainties. The first one is the uncertainty on the indicators of performance (which are not comparable) used by the decision maker to choose a solution (for example: service quality or inventory cost). Hence, we propose an approach based on subjective probability to evaluate the probability that a decision is optimal for the first actor and the probability that it is optimal for both. From these two evaluations, we propose a ranking function to help the first actor to take into account the second one when selecting a decision. The second kind of uncertainty pertains to the demand. A classical criterion under total uncertainty is Hurwicz criterion where a weight expresses a degree of pessimism. Nevertheless, the degree of pessimism is itself ill-known. Thus, it becomes difficult to take into account the behavior of the actors. Hence, we propose an approach based on possibility theory and the so-called pignistic transform, which computes a subjective probability distribution over the criteria. Then, we apply the method used for uncertain criterion. This approach is illustrated through an example and an industrial case study.
\end{abstract}

\section{Introduction}

In an increasingly competitive business world, where the sources of disturbance are drastically changing and increasing, supply chains actors are faced with the necessity to constantly improve their decision-making practices. The companies identifying supply chain risk as "an unavoidable and necessary task that continues to pose certain problems" (Lavastre et al., 2012). Faced with a "networked environment", "companies deepen their relationship with partners and thus become more dependent on each other" (Hallikas et al., 2005). Risk, in the context of an enterprise, is defined by Zsidisin (2003) as: "the danger that events or decisions will obstruct the company's achievement of its objectives". In this context, "The process of supply chain actors main can either amplify or absorb the effect of risks in the supply chain" (Juttner, 2005) when the principal risk comes from supply and

\footnotetext{
* Corresponding author.

E-mail addresses: guillaum@irit.fr (R. Guillaume),

gmarques@emse.fr (G. Marques), thierry@univ-tlse2.fr (C. Thierry), dubois@irit.fr (D. Dubois).
}

demand. Mastering the decision making processes of actors is therefore a key to minimizing the risks.

In this paper, we focus on the problem of designing the collaborative purchasing processes in the supply chain context under uncertainty. Moreover, we specifically consider a suppliercustomer relationship in a dyadic supply chain where actors are independent. This situation may be described as a 2-actor sequential decision problem. For an industrial Decision-Maker (DM) in a supply chain, the anticipation of the decisional behavior of his/her partners is common practice (capacity level, lot sizing rules, purchasing strategies, production scheduling...). He/she knows that his/her decision will be followed by a series of partner's decisions, which will impact the performance of his decision. The decisional behaviors of independent partners are extremely difficult to anticipate. These potential different behaviors can be interpreted as different sources of uncertainty for a particular actor of the chain.

In this paper, we deal with two sources of uncertain behavior. First, we consider the uncertainty of an actor about the performance criteria of the other actor (for instance one considers the inventory level whereas the other the service quality), criteria that are not commensurate. Moreover, we deal with behaviors under uncertainty 
(the pessimism or optimism of decision maker) which comes down of the occurrence of uncertain events (scraps, breakdowns, delays, demand fluctuations...) for which a probability distribution may not be accessible. Hence, in the context of uncertainty, the criterion should take into account the level of pessimism of decision makers.

This paper is organized in 7 sections. First, a literature review on supply chains is made (Section 2), then we provide some background on decision trees, possibility theory, pignistic probability, criteria under uncertainty and multi-actors decision that will be used in our proposal (Section 3). In the fourth section, we present our approach to model and support the decision-making process with one DM and then we propose a model for sequential multi-DM problem (Sections 5 and 6). Finally, Section 7 illustrates this proposal through an industrial case study.

\section{Literature review}

In our review, we distinguish the literature that focuses on minimizing risk in supply chains under uncertainty and the literature on coordination mechanisms inside the supply chain.

On the first topic, mostly optimization approaches have been proposed with a global supply chain optimization model and a single decision maker (Liang and Cheng, 2009; Peidro et al., 2009; Wang and Shu, 2005; Petrovic et al., 1999). Besides there exist robust optimization methods where suppliers share information with the customer (Guillaume et al., 2012, 2013). In these studies, the sources of uncertainty pertain to the demand, supply and process. Nevertheless, predefined criteria are used and the problem of distributed decision is not investigated, whereas it creates risks since the decisions made by suppliers impact decisions made by the customer.

Besides, the studies on coordination mechanisms focus on the minimization of sub-optimality of the distributed decision in the supply chain. Hence, an important part of the literature proposes and studies coordination mechanisms to obtain the optimal decision for the supply chain as a whole. For example, game theory is used for designing coordination mechanisms that may optimize the distributed system and studying coordination mechanisms as a form of cooperative advertising; see Aust and Buscher (2014) for a recent review. On their side, Chen, 2007, 2012 and Li et al., 2005 focus on procurement policy (price-only policies, quantity discount policy, etc.). Recently, Xiao et al. (2014) added the lead time as decision variable, on top of the price in context of make to order supply chains. Furthermore, the supply chains studied in this literature are mostly manufacturer-retailer supply chains, and these studies do not consider the planning process. Another part of this literature proposes coordination mechanism when the actors use linear mathematic models for production planning (Dudek and Stadtler, 2005) under perfect demand. In this part of the literature, the criterion of decision makers is predefined and the uncertainty is not taken into account. Moreover, in addition to this important academic research works, empirical analysis based on industrial case studies and decision-maker interviews have emphasized the fact that conceptual research has focused on the supply disruption risk with a little attention to the questions:

(i) "How views of supply disruption risk are developed and how these views affect the decision-making process" (Ellis et al., 2010),

(ii) What are supply chain managers' attitudes toward risk?

(iii) What are the ways in which decisions are made? (Lavastre et al., 2012)?

Moreover, Singh and Benyoucef (2013) emphasize the role of decision-making processes inside collaborative supply chains.
It shows the difficulty to establish decisions when confronted with conflicting individual interests and where "every company is responsible for its own risks and identifies the risks from its own viewpoint" (Hallikas et al., 2005).

\section{Background}

In this section, we recall formal tools we shall use to build the proposed approach.

\subsection{Tools for decision under imprecision}

In this section, we recall a model to represent the imprecision on the information (possibility distributions), how to derive a subjective probability from it (pignistic probability), a well-known criterion under total uncertainty.

\subsubsection{Possibility distributions}

Imprecise information is modeled by expressions of the form $v \in A$ where $A$ is a subset of $S$ that contains more than one element. Imprecision is always expressed by a disjunction of values (Dubois and Prade, 2009) that form a possibility distribution on $S$. The assertion $v \in A$ implies that all values from $v$ outside $A$ are supposed to be impossible.

A possibility distribution $\pi_{v}$ attached to an ill-known quantity $v$ quantifies the plausibility of values taken by $v$ (Dubois and Prade, 1988). It is a function from $S$ to a plausibility scale $L([0,1]$ for numerical possibility). A numerical possibility distribution taking a finite number of values $\lambda_{i} \in[0,1]$, for $i=1, \ldots, M$, may express imprecise probabilistic knowledge of the form $P\left(E_{i}\right) \geq 1-\lambda_{i}, i=1$,

...,M, where $E_{i}$ is a confidence set provided by the DM (Dubois and Prade, 2009). It can also be viewed as a random set $(m, F)_{\pi}$, with focal sets $E_{i}$ and masses $m\left(E_{i}\right)$, such that:

$$
\left\{\begin{array}{l}
E_{i}=\left\{x \in S \mid \pi(x) \geq \lambda_{i}\right\} \\
m\left(E_{i}\right)=\lambda_{i}-\lambda_{i-1}
\end{array}\right.
$$

The possibility distribution is then such that: $\pi(x)=\sum_{x \in E_{i}} m\left(E_{i}\right)$ (Dubois and Prade, 1982).

\subsubsection{Pignistic probability distribution}

The so-called pignistic probability extends Laplace principle of insufficient reason to possibility theory and to belief functions. It presupposes the idea that, while the knowledge or an actor can be too imprecise to be represented by a single probability distribution, the latter is needed when evaluating decisions in order to comply with the classical (Savage) decision theory (Smets, 2005). This probability distribution reflects betting odds used by the actor possessing a certain body of information. When the actor has no information, all alternatives are viewed as equally possible and the actor will bet on them at equal odds. Deriving the pignistic probability from a belief function consists in equally sharing the masses $(m, F)$ over each element of focal set $E$ for a random set $(m, F)$

$\operatorname{Pg}^{S}(x)=\sum_{E \subseteq S} \frac{m(E)}{|E|} \forall x \in S$

It can be viewed as the subjective probability distribution the decision-maker would provide via betting rates, had his knowledge been faithfully represented by the possibility distribution $\pi_{v}$. This probability distribution has been proposed by Dubois and Prade (1982) and axiomatized by Smets (2005), who coined it "pignistic". It coincides with the (older) Shapley value (Shapley, 1953 ) in the game theory. The pignistic probability distribution can be applied to possibility distributions and is also used in the simulation of "fuzzy variables" (Chanas and Nowakowski, 1988). 
Example 1. For example, let $\Pi\left(c^{1}\right)=1$ and $\Pi\left(c^{2}\right)=0.8$ be a possibility distribution over two possible criteria. It expresses the incomplete information that the probability that the actor uses criterion $c^{1}$ is at least 0.2 , while the other one is unknown. Let us compute the pignistic probability of each criterion. First, we compute the masses $m\left(E_{i}\right)$. In this case, the values of $\lambda_{i}$ are discrete values: $\lambda_{0}=0 ; \lambda_{1}=0.8 ; \lambda_{2}=1$. Then:

- $E_{1}=\left\{c^{1} ; c^{2}\right\}$ with $m\left(E_{1}\right)=0.8-0=0.8$

$-E_{2}=\left\{c^{1}\right\}$ with $m\left(E_{2}\right)=1-0.8=0.2$

From Eq. (6) we have: $\operatorname{Pg}\left(c^{1}\right)=\left(m\left(E_{1}\right) / 2\right)+\left(m\left(E_{2}\right) / 1\right)=(0.8 / 2)+$ $0.2=0.6$ and $\left(c^{2}\right)=\left(m\left(E_{1}\right) / 2\right)=(0.8 / 2)=0.4$.

While in the finite case, letting the DM directly provide such subjective probability degrees may be possible, it is too difficult for a DM to provide precise continuous subjective probability. In that case, it is more user-friendly to ask for weak information (like support and mode, or confidence intervals), to represent it faithfully in possibility theory, and then to extract the pignistic probability from it.

\subsubsection{Hurwicz criterion}

Hurwicz (1951) criterion for decision under total uncertainty is a parametric criterion which takes into account the optimism of decision maker. Let $\mathbb{D}=\left\{d_{1}, . ., d_{n}\right\}$ be the set of possible decisions, $S$ the set of possible states of the world, $X$ the set of the potential consequences of the decisions, $f_{d_{i}}(s) \in X$ the function defined for each decision $d_{i} \in \mathbb{D}$ which associates to each state $s \in S$ a precise consequence $x \in X, u\left(f_{d}(s)\right) \in \mathbb{R}$ the utility function that attaches a value to each consequence $x \in X$ and $\alpha$ the degree of pessimism of decision maker. The Hurwicz criterion achives a trade-off between the most cautious and the most risk evaluations:

$$
\begin{aligned}
& H_{u}\left(f_{d}\right)=\alpha \times \min _{s \in S}\left(u\left(f_{d}(s)\right)\right)+(1-\alpha) \times \max _{s \in S}\left(u\left(f_{d}(s)\right)\right) \\
& d^{*}=\underset{d \in \mathbb{D}}{\operatorname{argmax}}\left(H_{u}\left(f_{d}\right)\right)
\end{aligned}
$$

\subsection{Tools for multi-actors decision making}

In this section, we recall the model of sequential decision more precisely, namely decision trees and an egalitarist approach to multifactorial ranking of decisions: leximin.

\subsubsection{Decision tree}

In a real dynamic situation, the DM does not make a single decision, but a sequence thereof, according to the successive arrivals of relevant pieces of information. This type of problem is called dynamic decision under uncertainty. The decision made at time $t$ depends on the information available at $t$. By hypothesis, the information known at time $t$ is still known at time $t+\Delta t$. The incoming information is commonly viewed as "events". They are the results of an external independent entity, for example nature. In such conditions, we can call $\beta_{t}=\left\{e_{t}^{1}, \ldots, e_{t}^{m}\right\}$ and $\beta_{t+1}=$ $\left\{e_{t+1}^{1}, \ldots, e_{t+1}^{n}\right\}$ the sets of mutually exclusive and exhaustive events at time $t$ and $t+1$. The set $\beta_{t+1}$ refines a partition of states induced by the set $\beta_{t}$. We call $\mathbb{D}=\left\{D_{1}, \ldots, D_{T}\right\}$ the set of decisions that have been made at various times, where decision $D_{t}$ is made at time $t$.

This kind of problem has motivated many research works especially in the Artificial Intelligence literature (Nielsen and Jaffray, 2006; Jeantet et al., 2008; Jaffray and Jeleva, 2008). They are relevant in situations where a DM has a sequence of decisions (at prescribed times) to make. In this context, a strategy, called $\Delta$, is defined as a particular choice of decisions (one decision per possible situation). The set of all strategies is denoted by $\bar{\Delta}$. The target is therefore to support the DM who must choose the best strategy, $\Delta^{*}=\operatorname{argmax}(u(\Delta))$. All decisions are known when the $\Delta \in \bar{\Delta}$

strategy is applied.

A Decision Tree (DT) is often used to represent this kind of decisions. A DT may be defined as a directed acyclic graph $T=(\mathcal{N}, \mathscr{E})$ where $\mathcal{N}$ is the set of nodes and $\varepsilon$ the set of arcs and there exists a unique node (root node), from which there is a single path leading to any other node. The set of nodes is made of (Nielsen and Jaffray, 2006):

$-\mathcal{N}_{D}$ : the set of decision nodes (represented by squares). They characterize states where the DM has to decide and to choose one alternative among several ones. Each output arc of a decision node represents an alternative (some $d \in \mathbb{D}$ );

- $\mathcal{N}_{c}$ : the set of chance (or event) nodes (represented by circles). Event nodes represent the sources of uncertainty in the problem, i.e. states of nature. Each output arc of an event node shows a possible state of the world after the event has occurred (some $e \in \mathbb{E}$ );

- $C$ : the set of terminal nodes (leaves). A leaf is defined as a node without children $(\operatorname{child}(N)=\varnothing, \forall N \in C)$ and represents a terminal state of the sequential decision problem (a final consequence). A utility value is associated to each terminal node $(u(N), \forall N \in C)$.

In a decision tree, a strategy $\Delta$ is therefore defined as a set of arcs: $\Delta=\left\{\left(N, N^{\prime}\right): N \in \mathcal{N}_{D^{\Delta}}, N^{\prime} \in \mathcal{N}^{\Delta}\right\} \subseteq \mathscr{E} \sqrt{a^{2}+b^{2}}$ where $\mathcal{N}_{D^{\Delta}}=\mathcal{N}_{D} \cap \mathcal{N}^{\Delta}$ and $\mathcal{N}^{\Delta} \subseteq \mathcal{N}$ is the set of nodes involved in the strategy $\Delta$, i.e. the set of nodes made of:

- The root node: $N_{r}$ (a decision by hypothesis);

- A unique child for each decision made according to the strategy, i.e. $N \in \mathcal{N}_{D^{\Delta}}$;

- All the children of an event node met in the strategy, i.e. $N \in \mathcal{N}_{c^{\Delta}}=\mathcal{N}_{c} \cap \mathcal{N}^{\Delta}$.

We call $\bar{\Delta}^{T}$, the set of strategies in a given DT, T. An example of DT is given on Fig. 1.

It represents a decision situation where a DM has to decide at node $D_{1}$, then the event $E_{1}$ will occur, after what a second decision at $D_{2}$ will be made followed by a last event $E_{2}$. Formally:

- $\mathcal{N}_{D}=\left\{D_{1}, D_{2}\right\}$, with $\mathbb{D}_{1}=\left\{d_{1}^{1}, d_{1}^{2}\right\}$ and $\mathbb{D}_{2}=\left\{d_{2}^{1}, d_{2}^{2}\right\} ;$

- $\mathcal{N}_{c}=\left\{E_{1}, E_{2}\right\}$, with $S_{1}=\left\{e_{1}^{1}, e_{1}^{2}\right\}$ and $S_{2}=\left\{e_{2}^{1}, e_{2}^{2}\right\}$;

$-\beta_{1}=\left\{e_{1}^{1}, e_{1}^{2}\right\}$ and $\beta_{2}=\left\{e_{1}^{1} \cap e_{2}^{1}, e_{1}^{1} \cap e_{2}^{2}, e_{1}^{2} \cap e_{2}^{1}, e_{1}^{2} \cap e_{2}^{2}\right\}$.

One example of strategy is pictured in Fig. 1 (in bold). Enumerating the strategies may become a very hard computational problem because of the complexity of the decision situation (the number of strategies increases exponentially). Different methods have been proposed to find the best strategy (Hammond, 1988; Machina, 1989; McClennen, 1990; Nielsen and Jaffray, 2006).

Considering a multiple actor sequential game, algorithms based on the Dynamic Programming principle, i.e., backward induction, have to be preferred to search and find (if they exist) equilibria in this kind of game (Cachon and Netessine, 2006).

\subsubsection{Leximin criterion}

In the next section we shall use the leximin criterion to build a ranking function taking into account the collective satisfaction of several decision-makers without favoring any of them.

Definition 1. (Barbera and Jackson, 1988) Let $u_{j}(\bar{d}), j \in\{1, \ldots, m\}$ be the levels of satisfaction of the DMj for decision $\bar{d}$, and $a \in[0,1]$, 


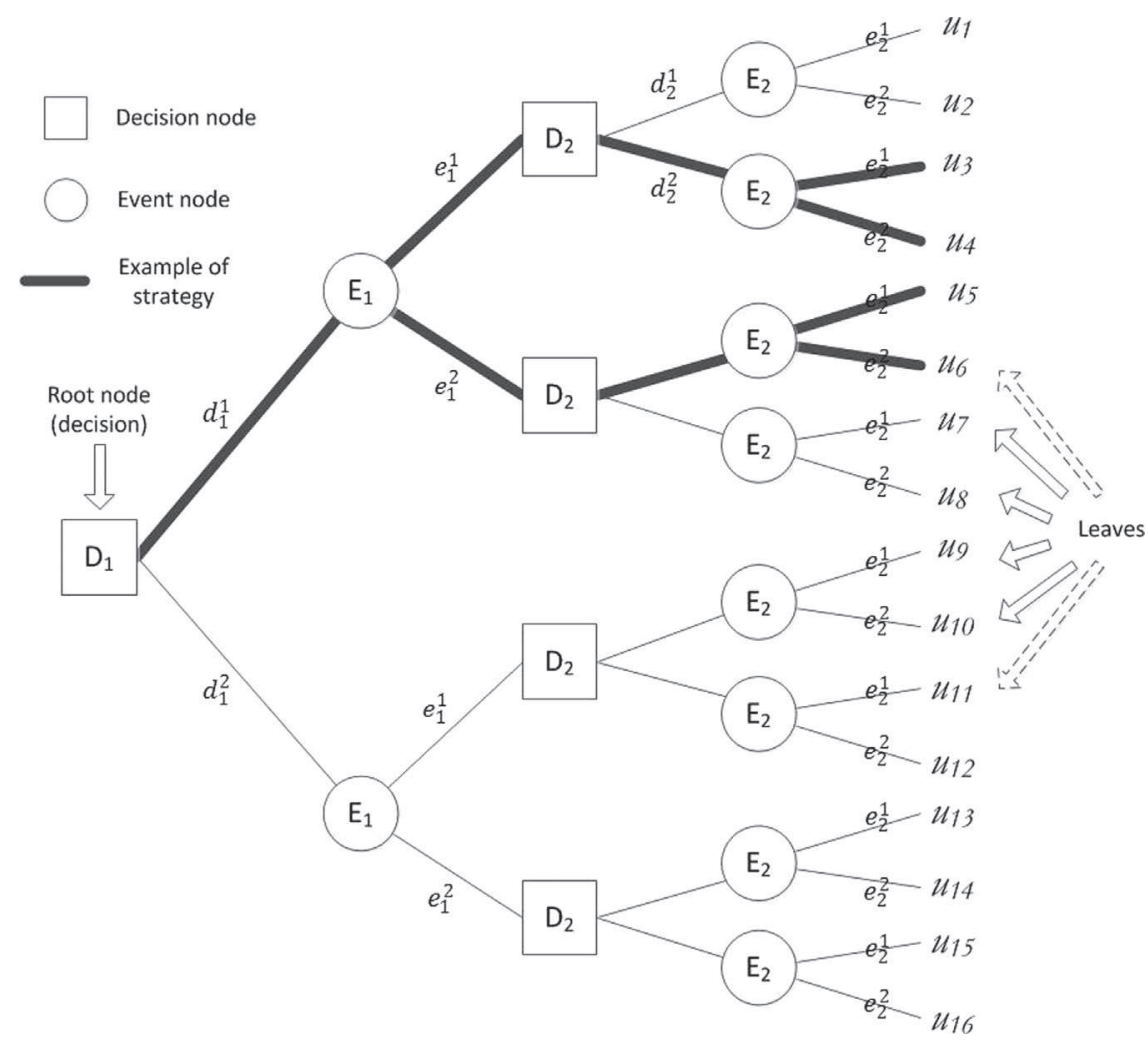

Fig. 1. Example of Decision Tree.

we define $J(a, \bar{d})=\left\{j \mid u_{j}(\bar{d}) \leq a\right\}$ and $U(a, \bar{d}) \mid$ the cardinality of $J(a, \bar{d})$. We write $\bar{u} \succ_{L m} \bar{v}$ if decision $\bar{u}$ is preferred to the decision $\bar{v}$ using the leximin criterion. The leximin is defined as follows (Eq. (5)): $\bar{u} \succ_{L m} \bar{v} \leftrightarrow \exists$ a such that $U(a, \bar{u})|<U(a, \bar{v})| \& \forall b<a U(b, \bar{u})|=U(b, \bar{v})|$

This criterion can also be defined by first reordering components of vectors $\bar{u}$ and as $\bar{v}$ in increasing order, and then ranking the two rearranged vectors in lexicographic order (see also Dubois et al., 1996). The leximin optimal decision can be interpreted in the context of multi-actor decision-making as an egalitarist solution.

\section{Ranking decisions taking into account uncertainty on criteria}

In this section, we present how a DM can make decision under uncertainty about his/her own criterion. Here we focus on the case where the criteria are not commensurable so an aggregation of ratings is not allowed. In this context, we suppose that the DM is satisfied if the decision $d \in D$ is optimal and not satisfied otherwise. More formally, the utility function of DM is:

$u(d)= \begin{cases}1 & \text { if decision } d \text { is optimal } \\ 0 & \text { otherewise }\end{cases}$

Moreover, we distinguish the case where the criteria are discrete (set of distinct criteria) and the case where the criterion has an imprecise parameter especially we focus on the case where the optimism degree of Hurwicz criterion is imprecise.

\subsection{Discrete case}

In the discrete case, we consider that the DM is able to give a subjective probability on each possible criterion $c \in C$ noted $\operatorname{Pg}(c)$.
The DM will take the decision that maximizes the expected value: $d^{*}=\operatorname{argmax}_{d \in D}\left(\sum_{c \in C} \operatorname{Pg}(c) u(d)\right)$.

Example 2. $C=\left\{c^{1}, c^{2}, c^{3}\right\}$ with the subjective probability $\operatorname{Pg}\left(c^{1}\right)=0.4, \operatorname{Pg}\left(c^{2}\right)=0.3$ and $\operatorname{Pg}\left(c^{2}\right)=0.3$ and the set of decisions $D=\left\{d^{1}, d^{2}, d^{3}\right\}$. Decisions $d^{1}$ and $d^{3}$ are both optimal for the criterion $c^{1}, d^{2}$ is optimal for criteria $c^{2}$ and $c^{3}$. So, the evaluation for each decision is: $0.4 \times 1+0.3 \times 0+0.3 \times 0=0.4$ for $d^{1}, 0.4 \times$ $0+0.3 \times 1+0.3 \times 1=0.6$ for $d^{2}$ and 0.4 for $d^{3}$. The optimal decision is $d^{2}$.

\subsection{Model of imprecise degree of optimism}

The model is based on the hypothesis that DM is able to give possibility distributions on the value of $\alpha$ : a possibility distribution $\tilde{\alpha}$ on his/her degree of optimism. Those possibility distributions can model vague adjectives like: pessimistic, optimistic, neutral (see Fig. 2(a)) or more precise as very pessimistic, little pessimistic, strongly neutral, (see Fig. 2(b)). Another way is to ask for the most plausible value of $\alpha$ and the maximal interval that contains $\alpha$. We can then build a triangular possibility distribution with these three values (see Fig. 2(c)).

Based on these possibility distributions and the study of change decision point (see Fig. 3), we can evaluate the stability of decisions (which in our approach comes down to computing the pignistic probability of being optimal). More precisely, we first build the pignistic probability distribution from the possibility distribution (cf Section 3.1.2), then compute the pignistic probability for each decision to be optimal. Namely, $\operatorname{Pg}(d=$ optimal $)=\operatorname{Pg}\left(\alpha \epsilon\left[\alpha_{d} ; \overline{\alpha_{d}}\right]\right)$ with $\left[\alpha_{d} ; \overline{\alpha_{d}}\right]$ the interval of values of $\alpha$ where $d$ is optimal. As before, DM will choose the decision that maximizes the expected value: $d^{*}=$ $\operatorname{argmax}_{d \in D}(\operatorname{Pg}(d=$ optimal $) u(d))$.

Example 3. consider two decisions $d^{1}$ and $d^{2}$ with respectively the minimal value equal 10 and 8 and maximal equal 25 and 30 . So the 



- - very optimistic $\quad$ - little optimistic $\quad$.... strongly neutral

Fig. 2. Choice of the possibility distribution.

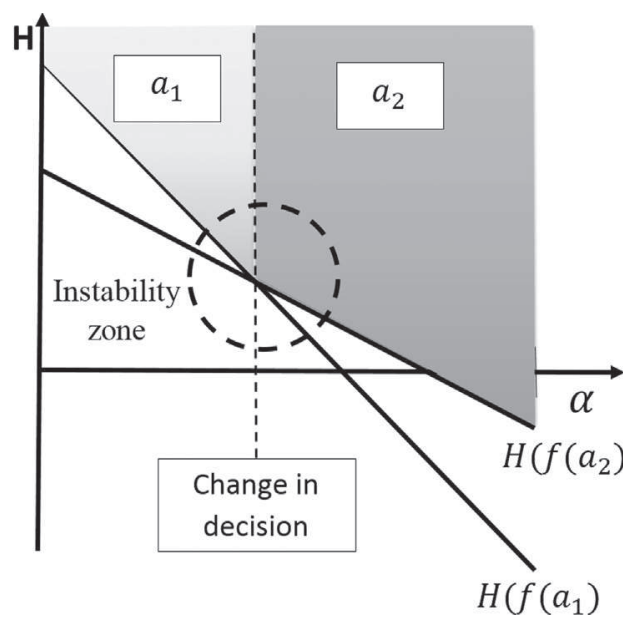

Fig. 3. Decision using Hurwicz criterion.

Hurwicz criteria of both decisions yield $H_{u}\left(d^{1}\right)=\alpha \times 10+(1-\alpha) \times$ 25 and $H_{u}\left(d^{2}\right)=\alpha \times 8+(1-\alpha) \times 30$ (see Fig. 4).

If $\alpha \in[(5 / 7) ; 1]$ the decision $d^{1}$ is optimal so $\left[\alpha_{d^{1}} ; \overline{\alpha_{d^{1}}}\right]=[(5 / 7) ; 1]$ and $\operatorname{Pg}(\alpha \epsilon[(5 / 7) ; 1]) \cong 0.9$

If $\alpha \in[0 ;(5 / 7)]$ the decision $d^{2}$ is optimal so $\left[\alpha_{d^{2}} ; \overline{\alpha_{d^{2}}}\right]=[0 ;(5 / 7)]$ and $\operatorname{Pg}(\alpha \epsilon[0 ;(5 / 7)]) \cong 0.1$.

Hence, the utility of DM for decision $d^{1}$ is 0.9 and 0.1 for $d^{2}$. Finally, DM chooses $d^{1}$.



Fig. 4. Decision under imprecise optimism degree.

\section{Sequential decision problem taking into account uncertainty on criteria}

In this section, we first introduce the main principles of our method of ranking decisions in a game with two players when (i) $\mathrm{DM}_{1}$ makes his decision before $\mathrm{DM}_{2}$ and (ii) $\mathrm{DM}_{1}$ only partially knows his/her own criterion and likewise for $\mathrm{DM}_{2}$ criterion. Moreover, we consider that under uncertainty on the criteria, the DM focuses on optimal decisions for each criterion. In other words, a decision may satisfy the DM if it is optimal for at least one criterion. Otherwise the decision is not considered.

We adopt the following notations:

- $C^{i}$ : set of criteria $c^{i}$ of $\mathrm{DM}_{i}$ with $i=1,2$

- $D^{1}$ : set of decisions $d^{1}$ of $\mathrm{DM}_{1}$ 
- $D_{j}^{2}$ : set of decisions $d_{j}^{2}$ of $\mathrm{DM}_{2}$ such that $\mathrm{DM}_{1}$ chooses decision $j$, $j$ being the index of decision node of $\mathrm{DM}_{1}$ with $j=1, \ldots, J$ and $J=\left|D^{1}\right|$

- $D=D_{1}^{2} \times \ldots \times D_{J}^{2}$ : set of decision vectors $\overrightarrow{d^{2}}=\left(d_{1}^{2}, \ldots, d_{J}^{2}\right)$ of $\mathrm{DM}_{2}$

$-C_{2}\left(\overrightarrow{d^{2}}\right)$ : set of criteria $c^{2} \in C^{2}$ for which decision vector $\overrightarrow{d^{2}}$ is optimal.

- $C_{1,2}\left(d^{1}, \overrightarrow{d^{2}}\right)$ : set of pairs of criteria $\left(c^{1}, c^{2}\right)$ for which $d^{1}$ is optimal for $c^{1} \in C^{1}$ and $d^{2}$ is optimal for $c^{2} \in C^{2}$.

- $C_{1}\left(d^{1}\right)$ : set of pairs of criteria $\left(c^{1}, c^{2}\right)$ for which decision $d^{1}$ is optimal

- $C_{2}^{1}\left(d^{1}\right)$ : set of criteria $c^{2} \in C^{2}$ for which decision $d^{1}$ is the best decision of $\mathrm{DM}_{1}$ for the $\mathrm{DM}_{2}$ using $c^{2}$

- $\operatorname{Pg}\left(c^{i}\right)$ : Pignistic probability of criterion $c^{i}$

- $\operatorname{Pg}\left(d^{1} \mid \mathrm{DM}_{1}\right)$ : is the pignistic probability that $d^{1}$ is an optimal decision from the point of view of $\mathrm{DM}_{1}$. The optimality of the decision depends on the criterion $c^{1}$ of $\mathrm{DM}_{1}$ and the criterion $c^{2}$ of $\mathrm{DM}_{2}$. It depends on the probability of using $c^{1}$ and $c^{2}$. So $\operatorname{Pg}\left(d^{1} \mid \mathrm{DM}_{1}\right)$ is the sum of the probabilities of pairs $\left(c^{1}, c^{2}\right)$ for which $d^{1}$ is optimal.

- $\operatorname{Pg}\left(d^{1} \mid \mathrm{DM}_{2}\right)$ : is the pignistic probability that $d^{1}$ is an optimal decision from the point of view of $\mathrm{DM}_{2}$. In other terms, the pignistic probability induced when the choice of $d^{1}$ is optimal for the $\mathrm{DM}_{2}$.

- $u_{i}(d)=\left\{\begin{array}{ll}1 & \text { if decision } d \text { is optimal } \\ 0 & \text { otherwise }\end{array}\right.$ the utility function for $\mathrm{DM}_{\mathrm{i}}$ with $d \in D^{i}$

\subsection{Overview of the approach}

To evaluate the probability that the decision $d$ is optimal in front of the possible criteria, we use the concept of pignistic probabilities (i.e. Section 3.1.2). Indeed the decision that has the maximal expected value to be optimal (using pignistic probability) is the one that is most likely to be optimal, taking into account the uncertainty on the criterion. Note that the expected value of $\mathrm{DM}_{\mathrm{i}}$, $E_{i}[d]$, with utility function $u_{i}(d)$, is equivalent to the pignistic probability that $d$ is an optimal decision from the point of view of $\mathrm{DM}_{i}: \operatorname{Pg}\left(d \mid \mathrm{DM}_{\mathrm{i}}\right)$. So in the next part of the paper we will use the notation $\operatorname{Pg}\left(d \mid \mathrm{DM}_{\mathrm{i}}\right)$ to refer to this expected value.

Since the decision of $\mathrm{DM}_{1}$ affects the possible decision of $\mathrm{DM}_{2}$, we propose to evaluate the decision of $\mathrm{DM}_{1}$ using the points of view of $\mathrm{DM}_{1}$ and $\mathrm{DM}_{2}$. Formally we assign a pair of values $\left(a_{d^{1}}, b_{d^{1}}\right)$ to each decision $d^{1} \in D^{1}$ where $a_{d^{1}}=\operatorname{Pg}\left(d^{1} \mid \mathrm{DM}_{1}\right)$ and $b_{d^{1}}=\operatorname{Pg}\left(d^{1} \mid \mathrm{DM}_{2}\right)$.

In order to compute the pignistic probability that decision is optimal, we have to know for which criteria this decision is optimal and then to sum the pignistic probabilities of these criteria. Moreover, we propose to rank the decisions using the importance given by $\mathrm{DM}_{1}$ to $\mathrm{DM}_{2}$. This importance is linked to the kind of relationship among $\mathrm{DM}_{1}$ and $\mathrm{DM}_{2}$. For instance $\mathrm{DM}_{1}$ will grant a high level of importance to her/his strategic or/and constraining partners whereas his/her non strategic and/or dependant partners will have a low level of importance (Marcotte et al., 2009).

\subsection{Evaluation of decisions under uncertain criteria}

The problem of computing the chance of optimality for $\mathrm{DM}_{1}$ and $\mathrm{DM}_{2}$ can be computed in 6 steps:

- Step 1. Computation of $C_{2}\left(\overrightarrow{d^{2}}\right)$ for each vector $\overrightarrow{d^{2}} \in D$, as follows:

$$
C_{2}\left(\overrightarrow{d^{2}}\right)=\left\{c^{2} \mid \overrightarrow{d^{2}} \text { is optimal }\right\}
$$

- Step 2. Computation of $\mathrm{C}_{1,2}\left(d^{1}, \overrightarrow{\mathrm{d}^{2}}\right)$ for each vector $\overrightarrow{\mathrm{d}^{2}} \in\left\{\overrightarrow{\mathrm{d}^{2}} \mid \mathrm{C}_{2}\left(\overrightarrow{\mathrm{d}^{2}}\right) \neq \varnothing\right\}$ and each decision $\mathrm{d}^{1} \in \mathrm{D}^{1}$, as follows:

$C_{1,2}\left(d^{1}, \overrightarrow{\mathrm{d}^{2}}\right)=\left\{\left(c^{1}, c^{2}\right) \mid d^{1}\right.$ and $\overrightarrow{\mathrm{d}^{2}}$ are optimal $\}$

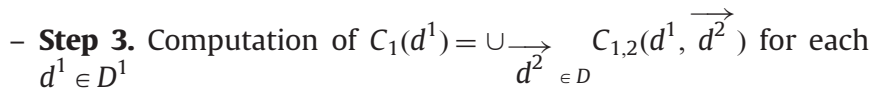

- Step 4. Computation of $\operatorname{Pg}\left(d^{1} \mid \mathrm{DM}_{1}\right)$ as follows:

$$
\operatorname{Pg}\left(d^{1} \mid \mathrm{DM}_{1}\right)=\sum_{\left(c^{1}, c^{2}\right) \in C_{1}\left(d^{1}\right)} \operatorname{Pg}\left(c^{1}\right) \times \operatorname{Pg}\left(c^{2}\right)
$$

- Step 5. Computation of $C_{2}^{1}\left(d^{1}\right)$ for each decision $d^{1} \in D^{1}$ as follows:

$$
C_{2}^{1}\left(d^{1}\right)=\left\{c^{2} \mid d^{1}=\operatorname{argmin}_{d^{1} \in D^{1}}\left(\min _{d^{2} \in D_{d^{1}}^{2}} c^{2}\left(d^{1}, d^{2}\right)\right)\right\}
$$

- Step 6. Computation of $\operatorname{Pg}\left(d^{1} \mid \mathrm{DM}_{2}\right)$ as follows:

$$
\operatorname{Pg}\left(d^{1} \mid \mathrm{DM}_{2}\right)=\sum_{c^{2} \in \mathrm{C}_{2}^{1}\left(d^{1}\right)} \operatorname{Pg}\left(c^{2}\right)
$$

5.3. Taking into account the importance given to the point of view of $\mathrm{DM}_{2}$

To help ssthe $\mathrm{DM}_{1}$ choose a decision, we propose to rank the possible optimal solutions according to the importance given to the point of view of $\mathrm{DM}_{2}$. If the importance given by $\mathrm{DM}_{1}$ to $\mathrm{DM}_{2}$ is very low, then she/he chooses the decision which maximizes her/his probability to be optimal for her/him. On the contrary, if the importance given by $\mathrm{DM}_{1}$ to $\mathrm{DM}_{2}$ is very high, then she/he chooses the decision which maximizes the probability for $\mathrm{DM}_{2}$. Between these extremes, different levels of importance will be considered. More formally let $f_{2}^{1}$ be the utility of a decision of $\mathrm{DM}_{1}$ from the point of view of $\mathrm{DM}_{2}$ (the latter being measured by $\operatorname{Pg}\left(d^{1} \mid \mathrm{DM}_{2}\right)$.

The function $f_{2}^{1}$ is a mapping $\operatorname{Pg}\left(d^{1} \mid \mathrm{DM}_{2}\right) \in[0 ; 1] \mapsto[-1 ; 1]$ that has to respect two requirements:

- If $\mathrm{DM}_{1}$ grants very low importance to $\mathrm{DM}_{2}$ :

$$
P g\left(d^{1} \mid \mathrm{DM}_{1}\right)>f_{2}^{1}\left(\operatorname{Pg}\left(d^{1} \mid \mathrm{DM}_{2}\right)\right), \quad \forall P g\left(d^{1} \mid \mathrm{DM}_{1}\right) \in[0 ; 1]
$$

- If $\mathrm{DM}_{1}$ grants the same importance to $\mathrm{DM}_{2}$ as to himself:

$$
P g\left(d^{1} \mid \mathrm{DM}_{2}\right)=f_{2}^{1}\left(\operatorname{Pg}\left(d^{1} \mid \mathrm{DM}_{2}\right)\right), \forall \operatorname{Pg}\left(d^{1} \mid \mathrm{DM}_{2}\right), \in[0 ; 1]
$$

To respect these characteristics, we propose that $f_{2}^{1}\left(\operatorname{Pg}\left(d^{1}\right)\right.$ $\left.\left.\mathrm{DM}_{2}\right)\right)=\operatorname{Pg}\left(d^{1} \mid \mathrm{DM}_{2}\right)+s_{2}^{1}$ with $s_{2}^{1} \in[-1 ; 1]$ such that if $s_{2}^{1}=1$ the importance given by $\mathrm{DM}_{1}$ to $\mathrm{DM}_{2}$ is very low otherwise if $s_{2}^{1}=-1$ then the importance given by $\mathrm{DM}_{1}$ to $\mathrm{DM}_{2}$ is very high.

In order to find the decision that ensures equity between the two decision makers $\mathrm{DM}_{1}$ and $\mathrm{DM}_{2}$ taking into account the importance given by $\mathrm{DM}_{1}$ to $\mathrm{DM}_{2}$, we propose to use the leximin criterion on the vector $\bar{v}=\left\langle\operatorname{Pg}\left(d^{1} \mid \mathrm{DM}_{1}\right) ; f_{2}^{1}\left(\operatorname{Pg}\left(d^{1} \mid \mathrm{DM}_{2}\right)\right)\right\rangle$. There are sevsseral cases:

- if $s_{2}^{1}=1$ (very low importance given by $\mathrm{DM}_{1}$ to $\mathrm{DM}_{2}$ ): we maximize first $\operatorname{Pg}\left(d^{1} \mid \mathrm{DM}_{1}\right)$ and then $\mathrm{f}_{2}^{1}\left(\operatorname{Pg}\left(d^{1} \mid \mathrm{DM}_{2}\right)\right)$ such that $\operatorname{Pg}\left(d^{1} \mid \mathrm{DM}_{1}\right) \leq \operatorname{Pg}\left(d^{1} \mid \mathrm{DM}_{2}\right)+1, \forall \operatorname{Pg}\left(d^{1} \mid \mathrm{DM}_{1}\right), \operatorname{Pg}\left(d^{1} \mid \mathrm{DM}_{2}\right) \in[0,1]$

- if $s_{2}^{1}=0$ (no difference between $\mathrm{DM}_{1}$ and $\mathrm{DM}_{2}$ ): we maximize indifferently the minimum between $\operatorname{Pg}\left(d^{1} \mid \mathrm{DM}_{1}\right)$ and $\operatorname{Pg}\left(d^{1} \mid\right.$ $\mathrm{DM}_{2}$ ) and the other 
- if $s_{2}^{1}=-1$ (very high importance given by $\mathrm{DM}_{1}$ to $\mathrm{DM}_{2}$ ): we maximize first $f_{2}^{1}\left(\operatorname{Pg}\left(d^{1} \mid \mathrm{DM}_{2}\right)\right)$ and then $\operatorname{Pg}\left(d^{1} \mid \mathrm{DM}_{1}\right)$ such that $\operatorname{Pg}\left(d^{1} \mid \mathrm{DM}_{2}\right)-1 \leq \operatorname{Pg}\left(d^{1} \mid \mathrm{DM}_{1}\right), \forall \operatorname{Pg}\left(d^{1} \mid \mathrm{DM}_{1}\right), \operatorname{Pg}\left(d^{1} \mid \mathrm{DM}_{2}\right) \in[0,1]$.

Note that we do not ask the decision-maker $\mathrm{DM}_{1}$ for parameter $s_{2}^{1}$. It is just an artifact of the method. By letting parameter $s_{2}^{1}$ range over its domain, we can explain whether a decision made by $\mathrm{DM}_{1}$ is likely to be accepted or not by $\mathrm{DM}_{2}$ in all situations. For instance:

- If $\forall s_{2}^{1} \in[-1 ; 1]$ optimal decision is the same then this decision will be accepted by the two DMs.

- If they exist an optimal decision $\left(d^{1 *}\right)$ for $s_{2}^{1} \in[-1 ; a]$ with $a$ close to -1 and other one $\left(d^{2 *}\right)$ for $s_{2}^{1} \in[a ; 1]$ the decision $d^{1 *}$ will be to be accepted by $\mathrm{DM}_{2}$.

\subsection{Example}

We illustrate the method in a general context, where $\mathrm{DM}_{1}$ does not know if $\mathrm{DM}_{2}$ will take him/her decision according to the minmax criterion (with probability 0.6 ) or Laplace's weighted average (with probability 0.4 ) on the indicator $f\left(d^{1}, d^{2}, n\right)$ and DM1 hesitates between the utility functions $g\left(d^{1}, d^{2}, n\right)$ (with probability 0.7 ) and $h\left(d^{1}, d^{2}, n\right)$ (with probability 0.3 ) within the minmax criteria:

$-C^{2}=\{$ minmax $;$ Laplace $\}$

$-C^{1}=\{g ; h\}$

\subsubsection{Evaluation of decision under uncertain criteria}

$\mathrm{DM}_{1}$ has 2 possible decisions $\{1 ; 2\}$ and $\mathrm{DM}_{2}$ has two possible decisions $\{$ one, two $\}$ and nature induces three possible realizations $\{a, b, c\}$. The evaluation of decision strategies is represented in Table 1 and Fig. 5.

To solve this problem, we apply the method presented in Section 5.2

- Step 1. Computation of $C_{2}\left(\overrightarrow{d^{2}}\right)$ for each vector $\overrightarrow{d^{2}} \in D$

We have 4 vectors $\overrightarrow{d^{2}}:\langle o n e$, one $\rangle,\langle$ one, two $\rangle,\langle t w o$, one $\rangle$,

$\langle t w o, t w o\rangle$. For this 4 vectors we compute $C_{2}\left(\overrightarrow{d^{2}}\right)$ :

1. $C_{2}(\langle$ one, one $\rangle)=\varnothing$

2. $C_{2}(\langle$ one,$t w o\rangle)=\{\operatorname{minmax}\}$

3. $C_{2}(\langle$ two, one $\rangle)=\{$ Laplace $\}$

4. $C_{2}(\langle t w o, t w o\rangle)=\varnothing$

- Step 2. Computation of $C_{1,2}\left(d^{1}, \overrightarrow{d^{2}}\right)$ for each vector $\overrightarrow{\mathrm{d}^{2}} \in\left\{D \mid C_{2}\left(\overrightarrow{\mathrm{d}^{2}}\right) \neq \varnothing\right\}$ and each $\mathrm{d}^{1} \in \mathrm{D}^{1}$

We have 4 combinations $\left(d^{1}, \overrightarrow{\mathrm{d}^{2}}\right)$ with $C_{2}\left(\overrightarrow{\mathrm{d}^{2}}\right) \neq \varnothing$

$(1,\langle$ one, two $\rangle),(2,\langle$ one,$t w o\rangle),(1,\langle t w o, o n e\rangle)$ and $(2,\langle t w o, o n e\rangle)$

For this 4 combinations we compute $C_{1,2}\left(d^{1}, \overrightarrow{d^{2}}\right)$ :

1. $\mathrm{C}_{1,2}(1,\langle$ one,$t w o\rangle)=\{(h ; \operatorname{minmax})\}$

Table 1

Evaluation of the decision strategies.

\begin{tabular}{|c|c|c|c|c|c|}
\hline \multirow[t]{2}{*}{$\mathrm{DM}_{1}$} & \multirow[t]{2}{*}{$\mathrm{DM}_{2}$} & \multicolumn{2}{|c|}{$f\left(d^{1}, d^{2}, n\right)$} & \multirow{2}{*}{$\frac{g\left(d^{1}, d^{2}, n\right)}{\max }$} & \multirow{2}{*}{$\frac{h\left(d^{1}, d^{2}, n\right)}{\max }$} \\
\hline & & $\max$ & Laplace & & \\
\hline \multirow[t]{2}{*}{1} & one & 10 & 8 & 10 & 12 \\
\hline & two & 14 & 7 & 11 & 10 \\
\hline \multirow[t]{2}{*}{2} & one & 20 & 10 & 14 & 11 \\
\hline & two & 15 & 12 & 9 & 15 \\
\hline
\end{tabular}



Fig. 5. DT of the example.

2. $\mathrm{C}_{1,2}(2,\langle$ one,$t w o\rangle)=\{(\mathrm{g} ;$ minmax $)\}$

3. $\mathrm{C}_{1,2}(1,\langle$ two, one $\rangle)=\{(\mathrm{g} ;$ Laplace $),(h ;$ Laplace $)\}$

4. $\mathrm{C}_{1,2}(2,\langle$ two, one $\rangle)=\varnothing$

- Step 3. Computation of $C_{1}\left(d^{1}\right)=\cup \underset{d^{2}}{\longrightarrow} C_{1,2}\left(d^{1}, \overrightarrow{d^{2}}\right)$ for each $d^{1} \in D^{1}$

1. $\mathrm{C}_{1}(1)=\left\{\begin{array}{c}(h ; \text { minmax }),(\mathrm{g} ; \text { Laplace }), \\ (h ; \text { Laplace })\end{array}\right\}$

2. $\mathrm{C}_{1}(2)=\{(\mathrm{g} ;$ minmax $)\}$

- Step 4. Computation of $\operatorname{Pg}\left(d^{1} \mid \mathrm{DM}_{1}\right)$

1. $\operatorname{Pg}\left(1 \mid \mathrm{DM}_{1}\right)=\sum_{c_{1}(1)} \operatorname{Pg}\left(c^{1}\right) \times P g\left(c^{2}\right)=0.3 \times 0.6+0.7 \times$ $0.4+0.3 \times 0.4=0.58$

2. $\operatorname{Pg}\left(2 \mid \mathrm{DM}_{1}\right)=\sum_{c_{1}(2)} \operatorname{Pg}\left(c^{1}\right) \times \operatorname{Pg}\left(c^{2}\right)=0.7 \times 0.6=0.42$

- Step 5. Computation of $C_{2}^{1}\left(d^{1}\right)$ for each decision $d^{1} \in D^{1}$

First we compute $\min _{d^{2} \in D_{d^{1}}^{2}} c^{2}\left(d^{1}, d^{2}\right)$ for $d^{1} \in D^{1}$ and $c^{2} \in C^{2}$

1. $\min _{d^{2} \in D_{1}^{2}} \max _{n \in N} f\left(1, d^{2}, n\right)=10$

2. $\min _{d^{2} \in D_{2}^{2}} \max _{n \in N} f\left(2, d^{2}, n\right)=15$

3. $\min _{d^{2} \in D_{1}^{2}} \sum_{n \in N} \frac{f\left(1, d^{2}, n\right)}{|N|}=7$

4. $\min _{d^{2} \in D_{2}^{2}} \sum_{n \in N} \frac{f\left(2, d^{2}, n\right)}{|N|}=10$

Then we compute:

1. $\operatorname{argmin}_{d^{1} \in D^{1}}\left(\min _{d^{2} \in D_{d^{1}}^{2}} \max _{n \in N} f\left(1, d^{2}, n\right)\right)$ $=\operatorname{argmin}\left(\min _{d^{2} \in D_{1}^{2}} \max _{n \in N} f\left(1, d^{2}, n\right) ; \min _{d^{2} \in D_{2}^{2}} \max _{n \in N} f\left(2, d^{2}, n\right)\right)$ $=\operatorname{argmin}(10 ; 15)=1$

2. $\operatorname{argmin}_{d^{1} \in D^{1}}\left(\min _{d^{2} \in D_{d^{1}}^{2}} \sum_{n \in N} \frac{f\left(1, d^{2}, n\right)}{|N|}\right)$

$$
\begin{aligned}
= & \operatorname{argmin}\left(\min _{d^{2} \in D_{1}^{2}} \sum_{n \in N} \frac{f\left(1, d^{2}, n\right)}{|N|} ;\right. \\
& \left.\min _{d^{2} \in D_{2}^{2}} \sum_{n \in N} \frac{f\left(2, d^{2}, n\right)}{|N|}\right) \\
= & \operatorname{argmin}(7 ; 10)=1
\end{aligned}
$$

From the previous results we build $C_{2}^{1}(1)$ and $C_{2}^{1}(2)$ :

1. $C_{2}^{1}(1)=\{\operatorname{minmax}$, Laplace $\}$

2. $C_{2}^{1}(2)=\varnothing$ 
Table 2

Results taking into account the importance given to the $\mathrm{DM}_{2}$.

\begin{tabular}{|c|c|c|c|c|c|c|c|c|c|c|c|c|c|c|c|c|c|c|c|c|c|}
\hline Optimal decision & $d=1$ & $d=1$ & $d=1$ & $d=1$ & $d=1$ & $d=1$ & $d=1$ & $d=1$ & $d=1$ & $d=1$ & $d=1$ & $d=1$ & $d=1$ & $d=1$ & $d=1$ & $d=1$ & $d=1$ & $d=1$ & $d=1$ & $d=1$ & $d=1$ \\
\hline$s_{2}^{1}$ & -1 & -0.9 & -0.8 & -0.7 & -0.6 & -0.5 & -0.4 & -0.3 & -0.2 & -0.1 & 0 & 0.1 & 0.2 & 0.3 & 0.4 & 0.5 & 0.6 & 0.7 & 0.8 & 0.9 & 1 \\
\hline$d=1$ & $\begin{array}{l}0 \\
0.58\end{array}$ & $\begin{array}{l}0.1 \\
0.58\end{array}$ & $\begin{array}{l}0.2 \\
0.58\end{array}$ & $\begin{array}{l}0.3 \\
0.58\end{array}$ & $\begin{array}{l}0.4 \\
0.58\end{array}$ & $\begin{array}{l}0.5 \\
0.58\end{array}$ & $\begin{array}{l}0.58 \\
0.6\end{array}$ & $\begin{array}{l}0.58 \\
0.7\end{array}$ & $\begin{array}{l}0.58 \\
0.8\end{array}$ & $\begin{array}{l}0.58 \\
0.9\end{array}$ & $\begin{array}{l}0.58 \\
1\end{array}$ & $\begin{array}{l}0.6 \\
1.1\end{array}$ & $\begin{array}{l}0.6 \\
1.2\end{array}$ & $\begin{array}{l}0.6 \\
1.3\end{array}$ & $\begin{array}{l}0.6 \\
1.4\end{array}$ & $\begin{array}{l}0.6 \\
1.5\end{array}$ & $\begin{array}{l}0.6 \\
1.6\end{array}$ & $\begin{array}{l}0.6 \\
1.7\end{array}$ & $\begin{array}{l}0.6 \\
1.8\end{array}$ & $\begin{array}{l}0.6 \\
1.9\end{array}$ & $\begin{array}{l}0.6 \\
2\end{array}$ \\
\hline$d=2$ & $\begin{array}{l}-1 \\
0.42\end{array}$ & $\begin{array}{c}-0.9 \\
0.42\end{array}$ & $\begin{array}{r}-0.8 \\
0.42\end{array}$ & $\begin{array}{c}-0.7 \\
0.42\end{array}$ & $\begin{array}{c}-0.6 \\
0.42\end{array}$ & $\begin{array}{c}-0.5 \\
0.42\end{array}$ & $\begin{array}{c}-0.4 \\
0.42\end{array}$ & $\begin{array}{c}-0.3 \\
0.42\end{array}$ & $\begin{array}{c}-0.2 \\
0.42\end{array}$ & $\begin{array}{c}-0.1 \\
0.42\end{array}$ & $\begin{array}{l}0 \\
0.42\end{array}$ & $\begin{array}{l}0.1 \\
0.4\end{array}$ & $\begin{array}{l}0.2 \\
0.4\end{array}$ & $\begin{array}{l}0.3 \\
0.4\end{array}$ & $\begin{array}{l}0.4 \\
0.4\end{array}$ & $\begin{array}{l}0.4 \\
0.5\end{array}$ & $\begin{array}{l}0.4 \\
0.6\end{array}$ & $\begin{array}{l}0.4 \\
0.7\end{array}$ & $\begin{array}{l}0.4 \\
0.8\end{array}$ & $\begin{array}{l}0.4 \\
0.9\end{array}$ & $\begin{array}{l}0.4 \\
1\end{array}$ \\
\hline
\end{tabular}

- Step 6. Computation of $\operatorname{Pg}\left(d^{1} \mid \mathrm{DM}_{2}\right)$

1. $\operatorname{Pg}\left(1 \mid \mathrm{DM}_{2}\right)=\sum_{c^{2} \in C_{2}^{1}(1)} \operatorname{Pg}\left(c^{2}\right)=0.7+0.3=1$

2. $\operatorname{Pg}\left(2 \mid \mathrm{DM}_{2}\right)=\sum_{c^{2} \in C_{2}^{1}(2)} \operatorname{Pg}\left(c^{2}\right)=0$

5.4.2. Ranking of decisions taking into account the importance given to $\mathrm{DM}_{2}$

In this example, we have $\operatorname{Pg}\left(1 \mid \mathrm{DM}_{1}\right)=0.58 ; \operatorname{Pg}\left(2 \mid \mathrm{DM}_{1}\right)=0.42$; $\operatorname{Pg}\left(1 \mid \mathrm{DM}_{2}\right)=1$ and $\operatorname{Pg}\left(2 \mid \mathrm{DM}_{2}\right)=0$.

To help $\mathrm{DM}_{1}$ rank the decisions in terms of the importance given by $\mathrm{DM}_{1}$ to $\mathrm{DM}_{2}, s_{2}^{1}$ evolves from -1 (very low importance) to 1 (very high importance). In order to summarize the knowledge and therefore to visualize possible change in the decision-making, we collect information in a table (Table 2). On the first line, you find the optimal solution depending of the value of $s_{2}^{1}$ recalled in line two. Then we give for each decision $d^{1} \in D^{1}$ the vector rearranged in increasing order.

From Table $2, \mathrm{DM}_{1}$ chooses decision 1 whatever the importance he/she gives to $\mathrm{DM}_{2}$ (best likelihood to be optimal for both DMs).

\section{The case of imprecise optimism degrees}

In this section, we consider the case of Hurwicz criterion with imprecise value of optimism degree $\alpha$. We describe how to compute the sets $C_{2}\left(\overrightarrow{d^{2}}\right), C_{1,2}\left(d^{1}, \overrightarrow{d^{2}}\right)$ and $C_{2}^{1}\left(d^{1}\right)$, in this context. We detail some steps of this method when (i) both $\mathrm{DM}_{1}$ and $\mathrm{DM}_{2}$ criteria are Hurwicz criteria with respectively $\tilde{\alpha^{1}}$ and $\tilde{\alpha_{1}^{2}}$ the fuzzy optimism degree of this criterion.

\subsection{Determination of $C_{2}\left(\overrightarrow{d^{2}}\right)$ (step 1)}

In this section, we give the framework of the algorithm to compute $C_{2}\left(\overrightarrow{d^{2}}\right)$ :

- Step 1.1. Computation of the value of $\tilde{\alpha_{1}^{2}}$ for which decision $d_{j}^{2}$ changes, denoted by $\alpha_{\text {change }}^{2}$, for each node of decision of $\mathrm{DM}_{2}$, (cf: Fig.3)

- Step 1.2. Computation of the set of $\tilde{\alpha}_{1}^{2}$ such that vector $\overrightarrow{d^{2}}$ is optimal for $\mathrm{DM}_{2}: C_{2}\left(\tilde{d}^{2}\right)$

The maximal cardinality of $C_{2}\left(\tilde{d}^{2}\right)$ appears when all decisions are optimal for a given $\alpha^{2}$ and each $\alpha_{\text {change }}^{2}$ are different for each decision nodes of $\mathrm{DM}_{2}$. Thus, in the worst case, we have $\left|D^{1}\right| \times\left|D^{2}\right|$ sets $C_{2}\left(d^{2}\right)$.

6.2. Determination of $C_{1,2}\left(d^{1}, \overrightarrow{d^{2}}\right)($ step 2$)$

After determining all $C_{2}\left(\overrightarrow{d^{2}}\right)$, we compute the set $C_{1,2}\left(d^{1}, \overrightarrow{d^{2}}\right)$ for each $d^{1} \in D^{1}$. The outline of the algorithm is:

- Step 2.1. Computation of the value of $\alpha^{1}$ for which decision $d^{1}$ changes, denoted by $\alpha_{\text {change }}^{1}$, for each $d^{2}$ such that $C_{2}\left(d^{2}\right) \neq \varnothing$, (see Fig. 4 Section 4.2)
- Step 2.2. Computation of the set of $\alpha^{1}$ such that $\overrightarrow{d^{2}}$ is the optimal vector of $\mathrm{DM}_{2}$ and $d^{1}$ is the optimal decision of $\mathrm{DM}_{1}: C_{1,2}\left(d^{1}, \overrightarrow{d^{2}}\right)$

In the worst case, we must compute $\left|D_{1}\right|$ for each $C_{2}\left(\overrightarrow{d^{2}}\right) \neq \varnothing$ so at most $\left|D^{1}\right|^{2} \times\left|D^{2}\right| C_{1,2}\left(d^{1}, \overrightarrow{d^{2}}\right)$.

\subsection{Determination of $C_{2}^{1}\left(d^{1}\right)$ (step 5)}

In this section we present the algorithm to compute the set of criteria giving an optimal solution the decision $d^{1}$ of $\mathrm{DM}_{1}$. This algorithm the result of $C_{2}\left(\overrightarrow{d^{2}}\right)$

- Step 5.1. Computation, for each vector $\overrightarrow{d^{2}} \in C_{2}\left(\overrightarrow{d^{2}}\right)$, of the value of $\alpha^{2}$ for which decision $d^{1}$ changes, denoted by $\alpha_{\text {change }}^{1}$, for each $\overrightarrow{d^{2}}$ such that $C_{2}\left(\overrightarrow{d^{2}}\right) \neq \varnothing$, (see Fig. 4 Section 4.2)

- Step 5.2. Computation of the set of $\alpha^{1}$ such that $\overrightarrow{d^{2}}$ is optimal vector of $\mathrm{DM}_{2}$ and $d^{1}$ is optimal decision of $\mathrm{DM}_{1}: C_{1,2}\left(d^{1}, \overrightarrow{d^{2}}\right)$ using Eq. (11)

$C_{2}^{1}\left(d^{1}\right)=\left\{c^{2} \mid d^{1}=\operatorname{argmin}_{d^{1} \in D^{1}}\left(\min _{d^{2} \in D_{d^{1}}^{2}} u_{c^{2}}\left(d^{1}, d^{2}\right)\right)\right\}$

\section{Application of the method to an industrial case-study}

In this section, we apply the method on a real-life case based on a dyadic supply chain where the customer, a French worldwide dermo-cosmetic maker (Pierre Fabre Dermo-Cosmétique), has to choose a collaboration protocol ( 2 possibilities) with its packaging product supplier. According to the traditional collaboration protocol the customer has to release orders (a product, a quantity) and the supplier responds. A DM's decision variable is the order lead time (here 12,8 or 6 weeks). With the advanced collaboration protocol the customer commits on purchases associated to a family of products 8 weeks in advance (product family aggregation is related to supplier's set up considerations). Then, the customer releases delivery needs about the product 1 week in advance. A DM's decision lever is the minimal volume associated to the family engagement (here $50,000,100,000$ or 150,000 products).

\subsection{Problem modeling}

According to the notation defined in previous parts, we denote by $\mathrm{DM}_{1}$ the customer (PFDC) and by $\mathrm{DM}_{2}$ one of his packaging suppliers. Two sequential decisions have to be made:

- $\mathrm{DM}_{1}$ has to define the collaboration protocol and its parameter (6 possibilities) (Table 3 ),

- then, $\mathrm{DM}_{2}$ will define his lot sizing strategy (3 possibilities). 
Table 3

Decision protocols for DM1's decisions.

\begin{tabular}{lll}
\hline Notation & Protocol decision & Parameter decision \\
\hline 1 & Advanced collaboration & Low volume $(50,000)$ \\
2 & Advanced collaboration & Medium volume $(100,000)$ \\
3 & Advanced collaboration & High volume $(150,000)$ \\
4 & Basic order & Little order lead time $(6 \mathrm{w})$ \\
5 & Basic order & Medium order lead time $(8 \mathrm{w})$ \\
6 & Basic order & Big order lead time $(12 \mathrm{w})$ \\
\hline
\end{tabular}

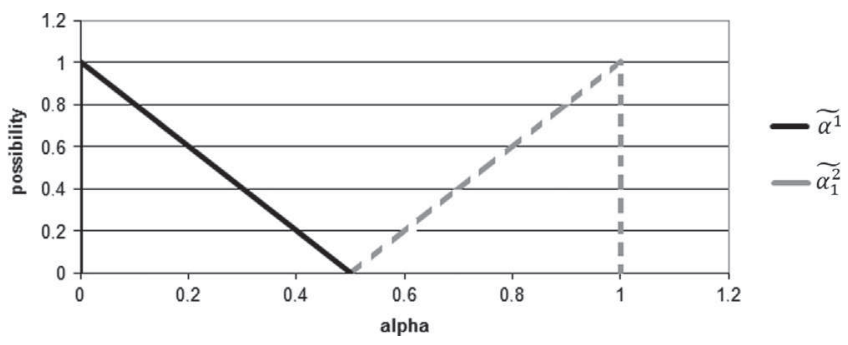

Fig. 6. Fuzzy optimism degree of $\mathrm{DM}_{1}$ and $\mathrm{DM}_{2}$.

In addition, the performance of the supply chain will be subject to a global uncertain event that models the uncertainty of the performance due to different risk sources (scrap, production/ transport delay, breakdowns...) (7 possible situations).

According to the number of scenarios that have to be evaluated, we use a simulation tool called LogiRisk for the evaluation of each scenario (each leaf of the tree) on real data from PFDC (Marques, 2010). Developed in the Perl language, it is dedicated to tactical and mostly strategic SC planning processes. This simulator is based on a discrete event simulation modeling approach. Authors have established a generic representation of the different planning processes for each SC actor based on the MRPII (Manufacturing Resource Planning) processes. An upstream planning process is used between partners: plans are made by the customer and passed to its suppliers. The procedure is repeated all over the chain in the upstream direction. No information circulates downstream (Lamothe et al., 2007, Marques et al., 2009).

The customer's cost function is $2 / 3$ average customer's stockout $1 / 3$ average customer's stock and supplier's cost function is $1 / 2$ average supplier's stock-out $1 / 2$ average supplier's inventory level.

\subsection{Problem solving}

The customer provides two possibility distributions on the optimism degree of himself/herself and on the supplier. The optimism degrees are represented in Fig. 6. Here, the $\mathrm{DM}_{1}$ is optimistic (black line) and the $\mathrm{DM}_{2}$ is known to be pessimistic (dotted line) by $\mathrm{DM}_{1}$.

From the simulation we build the decision tree (with 6 decisions for $\mathrm{DM}_{1}\{1 ; 2 ; 3 ; 4 ; 5 ; 6\}$ and 3 decisions for $\left.\mathrm{DM}_{2}\{1 ; 2 ; 3\}\right)$ and the cost function for each $\mathrm{DM}\left(\mathrm{DM}_{1}\right.$ : customer's cost and $\mathrm{DM}_{2}$ : supplier's cost).

\subsection{Determination of $C_{2}\left(\overrightarrow{d^{2}}\right)($ step 1$)$}

Decision 1 of $\mathrm{DM}_{2}$ is Pareto-optimal for all decisions of $\mathrm{DM}_{1}$. In other words, decision 1 has the minimal "min" and minimal "max" for each decision of $\mathrm{DM}_{1}$. So, whatever the optimism degree of
$\mathrm{DM}_{2}, \mathrm{DM}_{2}$ chooses decision 1 for each node.

$C_{2}((1,1,1,1,1,1))=[0.5 ; 1] \forall \overrightarrow{d^{2}} \neq(1,1,1,1,1,1) C_{2}\left(\overrightarrow{d^{2}}\right) \neq \varnothing$

7.4. Determination of $C_{1,2}\left(d^{1}, \overrightarrow{d^{2}}\right)($ step 2$)$

Then we compute the set $C_{1,2}\left(d^{1},(1,1,1,1,1,1)\right) \forall d^{1} \in D^{1}$. Whatever the optimism degree of $\mathrm{DM}_{1}$, decisions $1,4,5,6$ can be chosen:

$\forall d^{1} \neq 2,3 C_{1,2}\left(d^{1},(1,1,1,1,1,1)\right) \neq \varnothing$

$\mathrm{DM}_{1}$ has two possible optimal solutions: solution 2 and 3 .

So we compute $\alpha_{\text {change }}^{1}$ (Fig. 7): $C_{1,2}(2,(1,1,1,1,1,1))=[0 ; 0.429]$ and $C_{1,2}(3,(1,1,1,1,1,1))=[0.429 ; 0.5]$.

7.5. Computation of $C_{1}\left(d^{1}\right)$ (step 3$)$

In this example: $C_{1}\left(d^{1}\right)=C_{12}\left(d^{1},(1,1,1,1,1,1)\right) \forall d^{1} \in D^{1}$ :

$C_{1}(2)=C_{12}(2,(1,1,1,1,1,1))=[0 ; 0.429]$

$C_{1}(3)=C_{12}(3,(1,1,1,1,1,1))=[0.429 ; 0.5]$

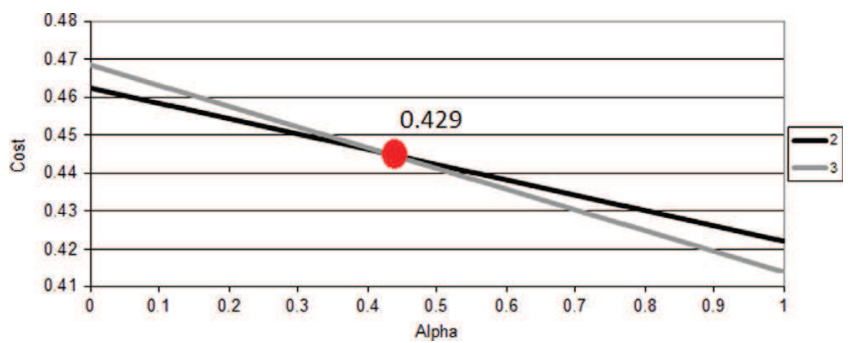

Fig. 7. Comparative analysis of decisions 2 and 3.

Table 4

Data of decision tree of the case-study.

\begin{tabular}{|c|c|c|c|c|c|}
\hline \multirow[t]{2}{*}{$\mathrm{DM}_{1}$} & \multirow[t]{2}{*}{$\mathrm{DM}_{2}$} & \multicolumn{2}{|c|}{ Supplier's cost } & \multicolumn{2}{|c|}{ Customer's cost } \\
\hline & & $\min$ & $\max$ & $\min$ & $\max$ \\
\hline \multirow[t]{3}{*}{1} & 1 & 7.175 & 7.696 & 0.471 & 0.537 \\
\hline & 2 & 14.516 & 17.563 & 0.415 & 0.475 \\
\hline & 3 & 20.436 & 25.396 & 0.411 & 0.453 \\
\hline \multirow[t]{3}{*}{2} & 1 & 6.022 & 6.907 & 0.422 & 0.462 \\
\hline & 2 & 13.078 & 14.34 & 0.380 & 0.425 \\
\hline & 3 & 18.92 & 21.57 & 0.375 & 0.414 \\
\hline \multirow[t]{3}{*}{3} & 1 & 5.905 & 6.956 & 0.414 & 0.468 \\
\hline & 2 & 12.975 & 14.734 & 0.382 & 0.420 \\
\hline & 3 & 18.267 & 21.257 & 0.374 & 0.412 \\
\hline \multirow[t]{3}{*}{4} & 1 & 6.177 & 7.272 & 0.547 & 0.656 \\
\hline & 2 & 11.862 & 14.444 & 0.505 & 0.605 \\
\hline & 3 & 17.268 & 20.824 & 0.478 & 0.554 \\
\hline \multirow[t]{3}{*}{5} & 1 & 6.427 & 6.946 & 0.571 & 0.622 \\
\hline & 2 & 12.131 & 13.985 & 0.567 & 0.624 \\
\hline & 3 & 17.540 & 20.445 & 0.542 & 0.639 \\
\hline \multirow[t]{3}{*}{6} & 1 & 7.307 & 7.549 & 0.765 & 1.009 \\
\hline & 2 & 13.010 & 14.628 & 0.763 & 1.009 \\
\hline & 3 & 18.968 & 21.294 & 0.765 & 1.008 \\
\hline
\end{tabular}

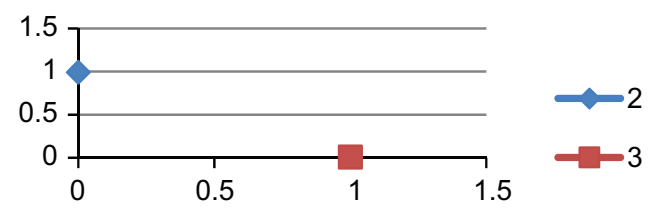

Fig. 8. Conflict of decisions. 
Table 5

Results taking into account the importance given to $\mathrm{DM}_{2}$.

\begin{tabular}{|c|c|c|c|c|c|c|c|c|c|c|c|c|c|c|c|c|c|c|c|c|c|}
\hline Optimal decision & $d=3$ & $d=3$ & $d=3$ & $d=3$ & $d=3$ & $d=3$ & $d=3$ & $d=3$ & $d=3$ & $d=3$ & $d=3$ & $d=2$ & $d=2$ & $d=2$ & $d=2$ & $d=2$ & $d=2$ & $d=2$ & $d=2$ & $d=2$ & $d=2$ \\
\hline $\boldsymbol{s}_{2}^{1}$ & -1 & -0.9 & -0.8 & -0.7 & -0.6 & -0.5 & -0.4 & -0.3 & -0.2 & -0.1 & 0 & 0.1 & 0.2 & 0.3 & 0.4 & 0.5 & 0.6 & 0.7 & 0.8 & 0.9 & 1 \\
\hline$d=2$ & -1 & $-0,9$ & $-0 ., 8$ & -0.7 & -0.6 & -0.5 & -0.4 & -0.3 & -0.2 & -0.1 & 0 & 0.1 & 0.2 & 0.3 & 0.4 & 0.5 & 0.6 & 0.7 & 0.8 & 0.9 & 1 \\
\hline & 1 & 1 & 1 & 1 & 1 & 1 & 1 & 1 & 1 & 1 & 1 & 1 & 1 & 1 & 1 & 1 & 1 & 1 & 1 & 1 & 1 \\
\hline \multirow[t]{2}{*}{$d=3$} & 0 & 0.02 & 0.02 & 0.02 & 0.02 & 0,02 & 0.02 & 0.02 & 0.02 & 0.02 & 0.02 & 0 & 0 & 0 & 0 & 0 & 0 & 0 & 0 & 0 & 0 \\
\hline & 0.84 & 0.84 & 0.84 & 0.84 & 0.84 & 0,84 & 0.84 & 0.84 & 0.84 & 0.9 & 1 & 1.1 & 1.2 & 1.3 & 1.4 & 1.5 & 1.6 & 1.7 & 1.8 & 1.9 & 2 \\
\hline
\end{tabular}

\subsection{Computation of $\operatorname{Pg}\left(d^{1} \mid D M_{1}\right)$ (step 4)}

To choose between decisions 2 and 3 we compute the pignistic probability that decision 2 is optimal: $\operatorname{Pg}\left(2 \mid \mathrm{DM}_{1}\right)=\operatorname{Pg}\left(\alpha^{1} \in\right.$ $[0 ; 0.429]) \times \operatorname{Pg}\left(\alpha^{2} \in[0 ; 1]\right) \cong 0.992 \times 1$ and the pignistic probability that decision 3 is optimal: $\operatorname{Pg}\left(3 \mid \mathrm{DM}_{1}\right)=\operatorname{Pg}\left(\alpha^{1} \in[0.429 ; 0.5]\right) \times$ $\operatorname{Pg}\left(\alpha^{2} \in[0 ; 1]\right) \cong 0.008 \times 1=0.008$ (Table 4).

\subsection{Determination of $C_{2}^{1}\left(d^{1}\right)$ (step 5)}

We compute $C_{2}^{1}\left(d^{1}\right)$ for 1 to 6 . Noted that the decision 1,4 , 5 and 6 are dominated by decision 2 and 3. But decision 3 is optimal for $\alpha^{2} \in[0.5 ; 1]$. So we deduce that $C_{2}^{1}(1)=C_{2}^{1}(2)=$ $C_{2}^{1}(4)=C_{2}^{1}(5)=C_{2}^{1}(6)=\varnothing$, and $C_{2}^{1}(3)=[0.5 ; 1]$.

\subsection{Computation of $\operatorname{Pg}\left(d^{1} \mid D M_{2}\right)$ (step 6)}

So, $\operatorname{Pg}\left(1 \mid \mathrm{DM}_{2}\right)=\operatorname{Pg}\left(2 \mid \mathrm{DM}_{2}\right)=0$ and $\operatorname{Pg}\left(3 \mid \mathrm{DM}_{2}\right)=1$.

A graphical representation of the resulting conflict of decisions (step 7) is pictured (Fig. 8):

Based on the evaluation of the pignistic probability of each decision of $\mathrm{DM}_{1}$ from the point of view of $\mathrm{DM}_{1}$ and $\mathrm{DM}_{2}$ we compute the optimal solution taking into account the importance given by $\mathrm{DM}_{1}$ to $\mathrm{DM}_{2}$ (Table 5).

In this case, the final decision depends on the balance of power between actors. Table 5 shows that the decision chosen by $\mathrm{DM}_{1}$ strongly depends on the importance given by $\mathrm{DM}_{1}$ to $\mathrm{DM}_{2}$. In fact, if $\mathrm{DM}_{1}$ gives low importance to $\mathrm{DM}_{2}\left(s_{2}^{1}>0,1\right)$ she/he imposes decision 2 to $\mathrm{DM}_{2}$ otherwise $\mathrm{DM}_{1}$ will choose decision 3 to satisfy $\mathrm{DM}_{2}$.

\section{Conclusion}

In this paper we focused on a decision problem in a dyadic collaborative supply chain. More precisely we addressed the problem of decision making for a customer, taking into account the future decision of his supplier under imprecise information on the criteria of the two SC partners. We proposed a decision method ensuring optimal stability. In other words we focus on the decision that has the best chance to be optimal under an imprecise criterion.

Industrial DMs are daily faced to the issue of exploiting their empirical knowledge of their partners' decisional behavior. This knowledge is rarely precise and quantified. Being able to exploit this knowledge may be a strategic advantage in term of value creation and preservation. The model presented in this paper and the associated case study illustrate the advantage to identify the decision which has the best chance of being optimal under imprecise knowledge, even if research efforts have to be made to improve the robustness of the results and to use real life collaboration experience in order to improve the mutual knowledge of partners' decisional behaviors.

\section{References}

Aust, G., Buscher, U., 2014. Cooperative advertising models in supply chain management: review. Eur. J. Oper. Res. 234 (1), 1-14.

Barbera, S., Jackson, M., 1988. Maximin, leximin and the protective criterion. J. Econ. Theory 46, 34-44.

Cachon, G.P., Netessine, S., 2006. Game theory in supply chain analysis. Tutor. Oper. Res.: Models, Methods, Appl. Innov. Decis. Mak., 200-233 (Chapter 8).

Chanas, S., Nowakowski, M., 1988. Single value simulation of fuzzy variable. Fuzzy Sets Syst. 122, 315-326.

Chen, F., 2007. Auction supply contracts. Manag. Sci. 53 (10), 1562-1576.

Chen, K., 2012. Procurement strategies and coordination mechanism of the supply chain with one manufacturer and multiple suppliers. Int. J. Prod. Econ. 138 (1), $125-135$.

Dubois, D., Fargier, H., Prade, H., 1996. Refinements of the maximin approach to decision-making in fuzzy environment. Fuzzy Sets Syst. 81, 103-122.

Dubois, D., Prade, H., 1982. On several representations of an uncertain body of evidence. In: Gupta, M., Sanchez, E. (Eds.), Fuzzy Information and Decision Processes. North-Holland, Amsterdam, pp. 167-181.

Dubois, D., Prade, H., 1988. PossibilityTheory: An Approach to ComputerizedProcessing of Uncertainty. Plenum Press, New York.

Dubois, D., Prade, H., 2009. In: Bouyssou, et al. (Eds.), Formal Representations of Uncertainty, pp. 85-156 (Chapter 3).

Dudek, G., Stadtler, H., 2005. Negotiation-based collaborative planning between supply chains partners. Eur. J. Oper. Res. 163 (3), 668-687, http://dx.doi.org/ 10.1016/j.ejor.2004.01.014.Abbecht 2010.

Ellis, S.C., Henry, R.M., Shockley, J., 2010. Buyer perceptions of supply disruption risk: a behavioral view and empirical assessment. J. Oper. Manag. 28, 34-46.

Guillaume, R., Grabot, B., Thierry, C., 2013. Management of the risk of backorders in a MTO-ATO/MTS context under imperfect requirements. Appl. Math. Model. 27 (16-17), 8060-8078.

Guillaume, R., Kobylanski, P., Zielinski, P., 2012. A robust lot sizing problem with illknown demands.. Fuzzy Sets Syst., 206; , pp. 39-57.

Hallikas, J., Karvonen, I., Pulkkinen, U., Virolainen, V.M., Tuominen, M., 2005. Risk management processes in supplier networks. Int. J. Prod. Econ. 90 (1), 47-58.

Hammond, P.J., 1988. Consequentialist foundations for expected utility. Theory Decis. 25, 25-78.

Hurwicz, L. 1951. Optimality criteria for decision making under ignorance. Cowles Commission Papers 370.

Jaffray, J.-Y., Jeleva, M., 2008. Information processing under imprecise risk with an insurance demand illustration. Int. J. Approx. Reason. 49 (1), 117-129.

Jeantet, G., P. Perny, O. Spanjaard, 2008. Rank-dependent probability weighting in sequential decision problems under uncertainty. In: Proceedings of the TwentySixth AAAI Conference on Artificial Intelligence, Chicago.

Juttner, U., 2005. Supply chain risk management: Understanding the business requirements from a practitioner perspective. Int. J. Logist. Manag. 16, 120-141.

Lamothe, J., Mahmoudi, J., Thierry, C., 2007. Cooperation to reduce risk in a telecom supply chain, special issue Managing Supply Chain Risk. Supply Chain Forum: Int. J. 8, 2.

Lavastre, O., Gunasekaran, A., Spalanzani, A., 2012. Supply chain risk management in French companies. Decis. Support Syst. 52 (4), 828-838.

Li, C., A. Ankolekar, S. Alan, S. Kaitia, 2005. Auctioning supply contracts with uncertain demand. Working paper, Graduate School of industrial Administration, Carnegis Mellon Univertsity.

Liang, T.F., Cheng, H.W., 2009. Application of fuzzy sets to manufacturing/distribution planning decisions with multi-product and multi-time period in supply chains. Expert Syst. Appl. 36, 3367-3377.

Machina, M.J., 1989. Dynamic consistency and non-expected utility models of choice under uncertainty. J. Econ. Lit. 27, 1622-1668.

Marcotte, F., Grabot, B., Costa Affonso, R., 2009. Cooperation models for Supply Chain Management. Int. J. Logist. Syst. Manag. 2 (19), 123-153.

Marques, 2010, Management des risques pour l'aide à la gestion de la collaboration au sein d'une chaîne logistique: une approche par simulation (Thèse de doctorat). INP Toulouse (in French).

Marques, G., Lamothe, J., Thierry, C., Gourc, D., 2009. A supply chain performance analysis of a pull inspired supply strategy faced to demand uncertainties. J. Intell. Manuf. (online).

McClennen, E.F., 1990. Rationality and dynamic choice: Foundational explorations. Cambridge Univ Press.

Nielsen, T.D., Jaffray, J.-Y., 2006. Dynamic decision making without expected utility: an operational approach. Eur. J. Oper. Res. 169 (1), 226-246. 
Peidro, D., Mula, J., Poler, R., Verdegay, J.L., 2009. Fuzzy optimization for supply chain planning under supply, demand and process uncertainties. Fuzzy Sets Syst. 160 (18), 2640-2657, http://dx.doi.org/10.1016/j.fss.2009.02.021.

Petrovic, D., Roy, R., Petrovic, R., 1999. Supply chain modelling using fuzzy sets. Int. J. Prod. Econ. 59, 443-453.

Shapley, L., 1953. A value for n-person games, Contributions to the Theory of Games. Editor: Harold W. Kuhn, Princeton University Press, pp. 307-317.

Singh, R.K., Benyoucef, L., 2013. A consensus based group decision making methodology for strategic selection problems of supply chain coordination. Eng. Appl. Artif. Intell. 26, 122-134.
Smets, P., 2005. Decision making in the TBM: the necessity of the pignistic transformation. Int. J. Approx. Reason. 38, 133-147.

Wang, J., Shu, Y.F., 2005. Fuzzy decision modeling for supply chain management. Fuzzy Sets Syst. 150, 107-127.

Xiao, T., Shi, J., Chen, G., 2014. Price and leadtime competition, and coordination for make-to-order supply chains. Comput. Ind. Eng. 68, 23-34.

Zsidisin, G.A., 2003. Managerial perceptions of supply risk. J. Supply Chain Manag. 39 (4), 14-26, http://dx.doi.org/10.1111/j.1745-493X.2003.tb00146.x. 